\title{
Hypergeometric solution of a certain polynomial Hamiltonian system of isomonodromy type
}

\author{
Teruhisa Tsuda \\ Faculty of Mathematics, Kyushu University, \\ Fukuoka 819-0395, Japan
}

April 30, 2010 (Revised: September 13, 2010)

\begin{abstract}
In [15] a unified description as polynomial Hamiltonian systems was established for a broad class of the Schlesinger systems including the sixth Painlevé equation and Garnier systems. The main purpose of this paper is to present particular solutions of this Hamiltonian system in terms of a certain generalization of Gauß's hypergeometric function. Key ingredients of the argument are the linear Pfaffian system derived from an integral representation of the hypergeometric function (with the aid of twisted de Rham theory) and Lax formalism of the Hamiltonian system.
\end{abstract}

\section{Introduction}

Fix integers $L \geq 2$ and $N \geq 1$. We consider the following completely integrable Hamiltonian system of partial differential equations:

$$
\frac{\partial q_{n}^{(i)}}{\partial x_{j}}=\frac{\partial H_{j}}{\partial p_{n}^{(i)}}, \quad \frac{\partial p_{n}^{(i)}}{\partial x_{j}}=-\frac{\partial H_{j}}{\partial q_{n}^{(i)}} \quad\left(\begin{array}{l}
i, j=1, \ldots, N \\
n=1, \ldots, L-1
\end{array}\right)
$$

with variables $x=\left(x_{1}, \ldots, x_{N}\right)$ and unknowns $q_{n}^{(i)}$ and $p_{n}^{(i)}$. Here the Hamiltonians function $H_{i}$ is given by

$$
x_{i} H_{i}=\sum_{n=0}^{L-1} e_{n} q_{n}^{(i)} p_{n}^{(i)}+\sum_{j=0}^{N} \sum_{0 \leq m<n \leq L-1} q_{m}^{(i)} p_{m}^{(j)} q_{n}^{(j)} p_{n}^{(i)}+\sum_{\substack{j=0 \\ j \neq i}}^{N} \frac{x_{j}}{x_{i}-x_{j}} \sum_{m, n=0}^{L-1} q_{m}^{(i)} p_{m}^{(j)} q_{n}^{(j)} p_{n}^{(i)}
$$

and

$$
x_{0}=q_{n}^{(0)}=q_{0}^{(i)}=1, \quad p_{n}^{(0)}=\kappa_{n}-\sum_{i=1}^{N} q_{n}^{(i)} p_{n}^{(i)}, \quad p_{0}^{(i)}=\theta_{i}-\sum_{n=1}^{L-1} q_{n}^{(i)} p_{n}^{(i)},
$$

2000 Mathematics Subject Classification 33C70, 34M55, 37K10.

Keywords: isomonodromic deformation, hypergeometric function, Painlevé equation, Schlesinger system.

E-mail: tudateru@math.kyushu-u.ac.jp 
thereby, $H_{i}$ forms a polynomial in the canonical variables. This system was introduced by the author in [15] via a similarity reduction of the UC hierarchy [14] (an extension of the KP hierarchy); it is equivalent to a class of the Schlesinger systems [11] describing isomonodromic deformations of an $L \times L$ Fuchsian system with $N+3$ poles on the Riemann sphere. The spectral type of this Fuchsian system is given by the $(N+3)$-tuple

$$
(1,1, \ldots, 1),(1,1, \ldots, 1), \underbrace{(L-1,1), \ldots,(L-1,1)}_{N+1}
$$

of partitions of $L$, which indicates how the characteristic exponents overlap at each of the $N+3$ regular singularities. System (1.1) contains complex constants

$$
(e, \kappa, \theta)=\left(e_{0}, e_{1}, \ldots, e_{L-1}, \kappa_{0}, \kappa_{1}, \ldots, \kappa_{L-1}, \theta_{0}, \theta_{1}, \ldots, \theta_{N}\right)
$$

satisfying the linear constraints

$$
\sum_{n=0}^{L-1} e_{n}=\frac{L-1}{2} \text { and } \sum_{n=0}^{L-1} \kappa_{n}=\sum_{i=0}^{N} \theta_{i}
$$

so the number of constant parameters is essentially $2 L+N-1$. Note that these parameters correspond to the characteristic exponents of the associated Fuchsian system. Henceforth, we denote by $\mathcal{H}_{L, N}$ the polynomial Hamiltonian system (1.1). For example, the case where $L=2$ and any $N \geq 1$ $\left(\mathcal{H}_{2, N}\right)$ coincides with the Garnier system in $N$ variables [5, 6] and, thus, the first nontrivial case $\left(\mathcal{H}_{2,1}\right)$ with the sixth Painlevé equation $P_{\mathrm{VI}}$ [8, 9]. For details refer to [15].

In this paper we present a family of particular solutions of the polynomial Hamiltonian system $\mathcal{H}_{L, N}$, which is parameterized by a point in the projective space $\mathbb{P}^{N(L-1)}$. These solutions are governed by a linear Pfaffian system of rank $N(L-1)+1$ and, furthermore, expressed in terms of a generalization of Gauß's hypergeometric function.

We begin by introducing the hypergeometric function crucial to this work. Fix the notation of multi-index; let $I=\left\{m=\left(m_{1}, \ldots, m_{N}\right) \mid m_{i} \in \mathbb{Z}_{\geq 0}\right\}$, and write $x^{m}=x_{1}{ }^{m_{1}} \cdots x_{N}{ }^{m_{N}}$ and $|m|=$ $m_{1}+\cdots+m_{N}$ for $m \in I$. We define a function $F_{L, N}=F_{L, N}(\alpha, \beta, \gamma ; x)$ in $N$ variables $x=\left(x_{1}, \ldots, x_{N}\right)$ by means of the power series

$$
F_{L, N}(\alpha, \beta, \gamma ; x) \stackrel{\text { def }}{=} \sum_{m \in I} \frac{\left(\alpha_{1}\right)_{|m|} \cdots\left(\alpha_{L-1}\right)_{|m|}\left(\beta_{1}\right)_{m_{1}} \cdots\left(\beta_{N}\right)_{m_{N}}}{\left(\gamma_{1}\right)_{|m|} \cdots\left(\gamma_{L-1}\right)_{|m|}(1)_{m_{1}} \cdots(1)_{m_{N}}} x^{m}
$$

convergent in the polydisc $\left\{\left|x_{1}\right|<1, \ldots,\left|x_{N}\right|<1\right\} \subset \mathbb{C}^{N}$. Here

$$
(\alpha, \beta, \gamma)=\left(\alpha_{1}, \ldots, \alpha_{L-1}, \beta_{1}, \ldots, \beta_{N}, \gamma_{1}, \ldots, \gamma_{L-1}\right)
$$

are complex constants such that $\gamma_{n} \notin \mathbb{Z}_{<0}$, and $(a)_{n}=\Gamma(a+n) / \Gamma(a)$. The series $F_{L, N}$ satisfies the system of linear differential equations

$$
\left\{x_{i}\left(\beta_{i}+\delta_{i}\right) \prod_{k=1}^{L-1}\left(\alpha_{k}+\mathcal{D}\right)-\delta_{i} \prod_{k=1}^{L-1}\left(\gamma_{k}-1+\mathcal{D}\right)\right\} y=0, \quad i=1, \ldots, N,
$$

where

$$
\delta_{i}=x_{i} \frac{\partial}{\partial x_{i}} \quad \text { and } \quad \mathcal{D}=\sum_{i=1}^{N} \delta_{i}
$$


As is shown in the next section, system (1.3) is equivalent to a linear Pfaffian system of rank $N(L-1)+1$. The holomorphic function $F_{L, N}(\alpha, \beta, \gamma ; x)$ at $0 \in \mathbb{C}^{N}$ can be analytically continued along any path on $X=\left\{x=\left(x_{1}, \ldots, x_{N}\right) \in \mathbb{C}^{N} \mid x_{i} \neq x_{j}(i \neq j), x_{i} \neq 0,1\right\}$, which is the complement of the singular locus of this Pfaffian system. Note that, if $(L, N)=(2,1),(L, 1)$ and $(2, N)$, then the hypergeometric series $F_{L, N}$ reduces to Gauß's ${ }_{2} F_{1}$, Thomae's ${ }_{L} F_{L-1}$ [13] and Appell-Lauricella's $F_{D}[2,7]$, respectively.

We turn now to the particular solutions of the Hamiltonian system $\mathcal{H}_{L, N}$. There is an equivalent formulation of $\mathcal{H}_{L, N}$ (Lax formalism) as a compatibility condition of an auxiliary linear problem consisting of the foregoing Fuchsian system and its deformation equations; see [15]. Under a certain condition of parameters we find particular solutions of $\mathcal{H}_{L, N}$ such that the associated Fuchsian system becomes reducible; furthermore, these solutions are governed by the same linear Pfaffian system of rank $N(L-1)+1$ as $F_{L, N}$. This fact leads us to the

Theorem 1.1. When $\kappa_{0}-\sum_{i=1}^{N} \theta_{i}=0$, the Hamiltonian system $\mathcal{H}_{L, N}$ possesses an $N(L-1)$-parameter family of particular solutions, each of which is expressed in terms of a hypergeometric function, i.e., an arbitrary solution of (1.3).

(See Theorem 3.2.)

Remark 1.2. The hypergeometric solutions of $P_{\mathrm{VI}}\left(=\mathcal{H}_{2,1}\right)$ and the Garnier system $\left(=\mathcal{H}_{2, N}\right)$ were first given by Fuchs [4] and Okamoto-Kimura [10], respectively. See also [5]. They linearized the Riccati-type equations for particular solutions by introducing new dependent variables, and then identified the resulting linear ones as the hypergeometric differential equations. Recently the case of $\mathcal{H}_{L, 1}$ was studied independently by Suzuki [12]; he obtained a power series solution through Frobenius' method after a direct linearization. The present result covers all previous ones; however, it is based on a method quite different from theirs. We emphasize that key ingredients of the argument are a systematic derivation of the linear Pfaffian system from an integral representation of the hypergeometric function (with the aid of twisted de Rham theory) and investigation into its Lax formalism rather than the Hamiltonian system itself.

In Sect. 2 we present the integral representation of the hypergeometric function $F_{L, N}$ (Proposition 2.1). Applying a twisted (co)homological technique we derive the linear Pfaffian system for $F_{L, N}$ (Theorem 2.2). In Sect. 3, after a brief review of Lax formalism of $\mathcal{H}_{L, N}$ (Theorem 3.1), we solve it with special values of parameters. Particular solutions thus obtained satisfy the same linear Pfaffian system as $F_{L, N}$. This establishes a representation of the solutions in terms of the hypergeometric functions (Theorem 3.2). In the appendix we summarize the contiguity relations for $F_{L, N}$.

\section{Integral representation and Pfaffian system for hypergeomet- ric function $F_{L, N}$}

In this section we first introduce the integral representation of $F_{L, N}$, then from which we derive the linear Pfaffian system by means of the viewpoint of twisted de Rham theory. Conversely, the hypergeometric function $F_{L, N}$ can be characterized as the unique holomorphic solution of this Pfaffian system at $0 \in \mathbb{C}^{N}$. 


\subsection{Integral representations}

The hypergeometric function $F_{L, N},(1.2)$, can be written as

$$
\begin{aligned}
& F_{L, N}(\alpha, \beta, \gamma ; x)=\prod_{k=1}^{L-1} \frac{\Gamma\left(\gamma_{k}\right)}{\Gamma\left(\alpha_{k}\right) \Gamma\left(\gamma_{k}-\alpha_{k}\right)} \\
& \quad \times \int_{[0,1]^{L-1}} \prod_{k=1}^{L-1} z_{k}^{\alpha_{k}-1}\left(1-z_{k}\right)^{\gamma_{k}-\alpha_{k}-1} \prod_{i=1}^{N}\left(1-x_{i} z_{1} z_{2} \cdots z_{L-1}\right)^{-\beta_{i}} \mathrm{~d} z_{1} \cdots \mathrm{d} z_{L-1},
\end{aligned}
$$

provided $\left|x_{i}\right|<1$ and $\operatorname{Re}\left(\gamma_{k}\right)>\operatorname{Re}\left(\alpha_{k}\right)>0$ and the branch of the integrand is assigned as

$$
\arg z_{k}=\arg \left(1-z_{k}\right)=0 \quad \text { and } \quad\left|\arg \left(1-x_{i} z_{1} z_{2} \cdots z_{L-1}\right)\right|<\frac{\pi}{2} .
$$

Representation (2.1) can be verified in a standard manner, i.e., by means of the binomial theorem:

$$
(1-z)^{-a}=\sum_{n=0}^{\infty} \frac{(a)_{n}}{(1)_{n}} z^{n}
$$

and the relation between the beta function and gamma function:

$$
B(a, b)=\int_{0}^{1} z^{a-1}(1-z)^{b-1} \mathrm{~d} z=\frac{\Gamma(a) \Gamma(b)}{\Gamma(a+b)}, \quad \operatorname{Re}(a), \operatorname{Re}(b)>0 .
$$

To transform the nonlinear form in the integrand of (2.1) into a linear one, we apply the change of integration variables

$$
t_{1}=z_{1}, \quad t_{2}=z_{1} z_{2}, \quad t_{3}=z_{1} z_{2} z_{3}, \quad \ldots, \quad t_{L-1}=z_{1} z_{2} \cdots z_{L-1} .
$$

The Jacobian of this transformation is calculated as

$$
\left|\frac{\partial\left(z_{1}, \ldots, z_{L-1}\right)}{\partial\left(t_{1}, \ldots, t_{L-1}\right)}\right|=\frac{1}{t_{1} t_{2} \cdots t_{L-2}} .
$$

Put $t_{0}=1$ for convenience. The integral representation of $F_{L, N}$ in which every factor of the integrand takes a linear form is, therefore, established.

Proposition 2.1. Assume $\operatorname{Re}\left(\gamma_{k}\right)>\operatorname{Re}\left(\alpha_{k}\right)>0$. For $\left|x_{i}\right|<1$ it holds that

$$
\begin{aligned}
& F_{L, N}(\alpha, \beta, \gamma ; x)=\prod_{k=1}^{L-1} \frac{\Gamma\left(\gamma_{k}\right)}{\Gamma\left(\alpha_{k}\right) \Gamma\left(\gamma_{k}-\alpha_{k}\right)} \\
& \quad \times \int_{\Delta} t_{L-1}{ }^{\alpha_{L-1}-1} \prod_{k=1}^{L-2} t_{k}^{\alpha_{k}-\gamma_{k+1}} \prod_{k=1}^{L-1}\left(t_{k-1}-t_{k}\right)^{\gamma_{k}-\alpha_{k}-1} \prod_{i=1}^{N}\left(1-x_{i} t_{L-1}\right)^{-\beta_{i}} \mathrm{~d} t_{1} \cdots \mathrm{d} t_{L-1}
\end{aligned}
$$

with the integration domain $\Delta$ being an $(L-1)$-simplex

$$
\Delta=\left\{0 \leq t_{L-1} \leq \cdots \leq t_{2} \leq t_{1} \leq 1\right\} \subset \mathbb{R}^{L-1} .
$$

Here the branch of the integrand is assigned as

$$
\arg t_{k}=\arg \left(t_{k-1}-t_{k}\right)=0 \quad \text { and } \quad\left|\arg \left(1-x_{i} t_{L-1}\right)\right|<\frac{\pi}{2}
$$

Based on this integral representation and twisted de Rham theory, we will discuss below the linear Pfaffian system characterizing $F_{L, N}$. 


\subsection{Pfaffian system}

Consider a multivalued function

$$
U(t)=t_{L-1}{ }^{\alpha_{L-1}} \prod_{k=1}^{L-2} t_{k}^{\alpha_{k}-\gamma_{k+1}} \prod_{k=1}^{L-1}\left(t_{k-1}-t_{k}\right)^{\gamma_{k}-\alpha_{k}} \prod_{i=1}^{N}\left(1-x_{i} t_{L-1}\right)^{-\beta_{i}}
$$

with $t_{0}=1$ defined on

$$
T=\left\{t=\left(t_{1}, \ldots, t_{L-1}\right) \in \mathbb{C}^{L-1} \mid t_{k} \neq 0, t_{k} \neq t_{k-1}, t_{L-1} \neq 1 / x_{i}\right\},
$$

which is the complement of singular locus $D=\bigcup_{k=1}^{L-1}\left(\left\{t_{k}=0\right\} \cup\left\{t_{k-1}-t_{k}=0\right\}\right) \cup \bigcup_{i=1}^{N}\left\{1-x_{i} t_{L-1}=\right.$ $0\}$ of $U(t)$ in $\mathbb{C}^{L-1}$. Let $\mathcal{L}$ be the local system of rank one determined by $1 / U(t)$, i.e., a flat line bundle consisting of the local solutions of $\nabla_{\omega} h=0$ on $T$, where $\nabla_{\omega}$ is the covariant differential operator given by

$$
\nabla_{\omega}=\mathrm{d}+\omega \wedge, \quad \omega=\mathrm{d} \log U(t) .
$$

Let $\mathcal{L}^{\vee}$ be the dual local system of $\mathcal{L}$. Denote by $H^{p}(T, \mathcal{L})\left(\operatorname{resp} . H_{p}\left(T, \mathcal{L}^{\vee}\right)\right)$ the $p$-th cohomology (resp. homology) group with coefficients in $\mathcal{L}$ (resp. $\mathcal{L}^{\vee}$ ). Under a certain genericity condition for the exponents $\alpha_{i}, \beta_{i}, \gamma_{i} \in \mathbb{C} \backslash \mathbb{Z}$, it holds that

$$
\operatorname{dim} H^{p}(T, \mathcal{L})=\operatorname{dim} H_{p}\left(T, \mathcal{L}^{\vee}\right)= \begin{cases}N(L-1)+1 & \text { if } p=L-1 \\ 0 & \text { if } p \neq L-1\end{cases}
$$

and, furthermore, bases of the top cohomology and homology groups are described as follows. First we notice the isomorphism

$$
\begin{aligned}
H^{p}(T, \mathcal{L}) & \cong H^{p}\left(\Omega^{\bullet}(* D), \nabla_{\omega}\right) \\
& =\left\{\xi \in \Omega^{p}(* D) \mid \nabla_{\omega} \xi=0\right\} / \nabla_{\omega} \Omega^{p-1}(* D),
\end{aligned}
$$

where the right-hand side is the de Rham cohomology group determined by $\nabla_{\omega}$, and $\Omega^{p}(* D)$ stands for the space of rational $p$-forms holomorphic outside $D$. It can be verified that the rational $(L-1)$ forms

$$
\begin{aligned}
\varphi_{0} & =\frac{\mathrm{d} t_{1} \wedge \cdots \wedge \mathrm{d} t_{L-1}}{t_{L-1} \prod_{k=1}^{L-1}\left(t_{k-1}-t_{k}\right)}, \\
\varphi_{n}^{(i)} & =\frac{\mathrm{d} t_{1} \wedge \cdots \wedge \mathrm{d} t_{L-1}}{t_{L-1}\left(1-x_{i} t_{L-1}\right) \prod_{\substack{k=1 \\
k \neq n}}^{L-1}\left(t_{k-1}-t_{k}\right)} \quad\left(\begin{array}{c}
1 \leq i \leq N \\
1 \leq n \leq L-1
\end{array}\right)
\end{aligned}
$$

are cocycles representing a basis of $H^{L-1}(T, \mathcal{L})$. On the other hand, a basis of $H_{L-1}\left(T, \mathcal{L}^{\vee}\right)$ can be constructed from the set of bounded chambers in the real locus $T \cap \mathbb{R}^{L-1}$ of $T$. For simplicity, we fix the configuration $x \in \mathbb{C}^{N}$ of $N$ points to be real numbers such that $0<x_{N}<\cdots<x_{2}<x_{1}<1$. Accordingly, the set of bounded chambers is given by

$$
\begin{aligned}
\Delta_{0}{ }^{\prime} & =\left\{0<t_{L-1}<\cdots<t_{1}<t_{0}=1\right\}, \\
\Delta_{n}^{(i)^{\prime}} & =\left\{\begin{array}{c}
t_{L-1}>t_{L-2}>\cdots>t_{L-n-1}>0 \\
0<t_{L-n-1}<\cdots<t_{1}<t_{0}=1 \\
1 / x_{i-1}<t_{L-1}<1 / x_{i}
\end{array}\right\} \quad\left(\begin{array}{c}
1 \leq i \leq N \\
1 \leq n \leq L-1
\end{array}\right) .
\end{aligned}
$$


The regularizations [1, 16] of these cycles, denoted by $\Delta_{0}$ and $\Delta_{n}^{(i)}$, represent a basis of $H_{L-1}\left(T, \mathcal{L}^{\vee}\right)$.

Now we introduce the integrals

$$
y_{0}=\int_{\Delta} U(t) \varphi_{0} \quad \text { and } \quad y_{n}^{(i)}=\int_{\Delta} U(t) \varphi_{n}^{(i)}
$$

for any twisted cycle $\Delta \in H_{L-1}\left(T, \mathcal{L}^{\vee}\right)$. Then we have the

Theorem 2.2. The functions $y_{0}$ and $y_{n}^{(i)}$ satisfy the linear Pfaffian system

$$
\begin{aligned}
\left(x_{i}-1\right) \frac{\partial y_{0}}{\partial x_{i}}= & \beta_{i}\left(-y_{0}+\sum_{m=1}^{L-1} y_{m}^{(i)}\right), \\
\left(x_{i}-x_{j}\right) \frac{\partial y_{n}^{(j)}}{\partial x_{i}}= & \beta_{i}\left(y_{n}^{(i)}-y_{n}^{(j)}\right), \\
x_{i} \frac{\partial y_{n}^{(i)}}{\partial x_{i}}= & -\alpha_{n} y_{n}^{(i)}+\left(\gamma_{n}-\alpha_{n}\right) \sum_{m=n+1}^{L-1} y_{m}^{(i)}+\frac{\gamma_{n}-\alpha_{n}}{x_{i}-1}\left(-y_{0}+\sum_{m=1}^{L-1} y_{m}^{(i)}\right) \\
& +\sum_{\substack{j=1 \\
j \neq i}}^{N} \frac{\beta_{j} x_{j}}{x_{i}-x_{j}}\left(y_{n}^{(j)}-y_{n}^{(i)}\right) .
\end{aligned}
$$

A proof of this theorem will be given in Sect. 2.3.

Let us consider the vector-valued function

$$
\vec{y}=\vec{y}(x ; \Delta)={ }^{\mathrm{T}}\left(y_{0}, y_{1}^{(1)}, y_{2}^{(1)}, \ldots, y_{L-1}^{(1)}, y_{1}^{(2)}, y_{2}^{(2)}, \ldots, y_{L-1}^{(2)}, \ldots, y_{1}^{(N)}, y_{2}^{(N)}, \ldots, y_{L-1}^{(N)}\right)
$$

defined by the integrals (2.4) for $\Delta \in H_{L-1}\left(T, \mathcal{L}^{\vee}\right)$. Then (2.5) takes the following expression:

$$
\mathrm{d} \vec{y}=\left\{\sum_{i=1}^{N}\left(E_{i} \mathrm{~d} \log x_{i}+F_{i} \mathrm{~d} \log \left(x_{i}-1\right)\right)+\sum_{1 \leq i<j \leq N} G_{i j} \mathrm{~d} \log \left(x_{i}-x_{j}\right)\right\} \vec{y},
$$

where $E_{i}, F_{i}$ and $G_{i j}$ are the square matrices of size $N(L-1)+1$ :

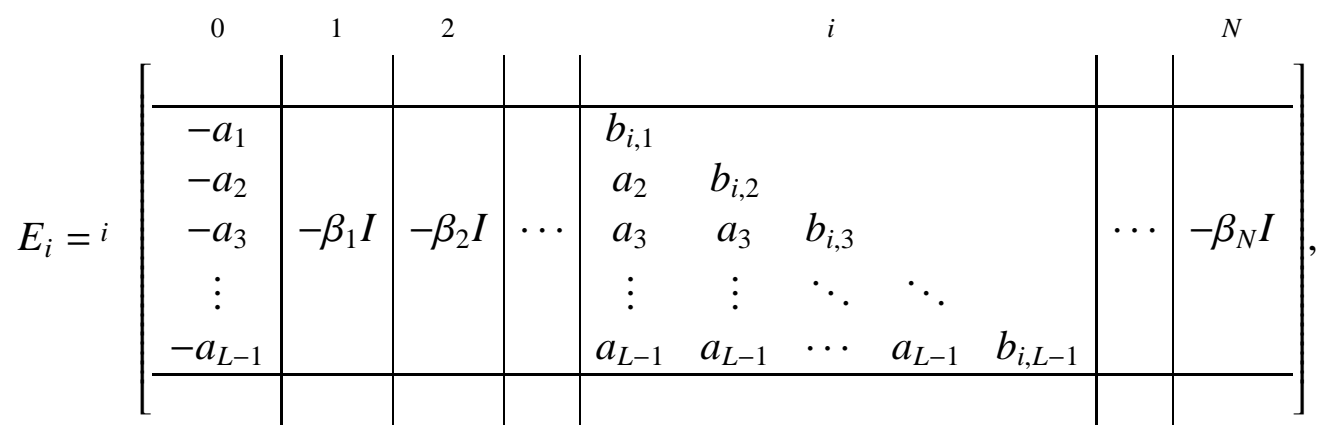

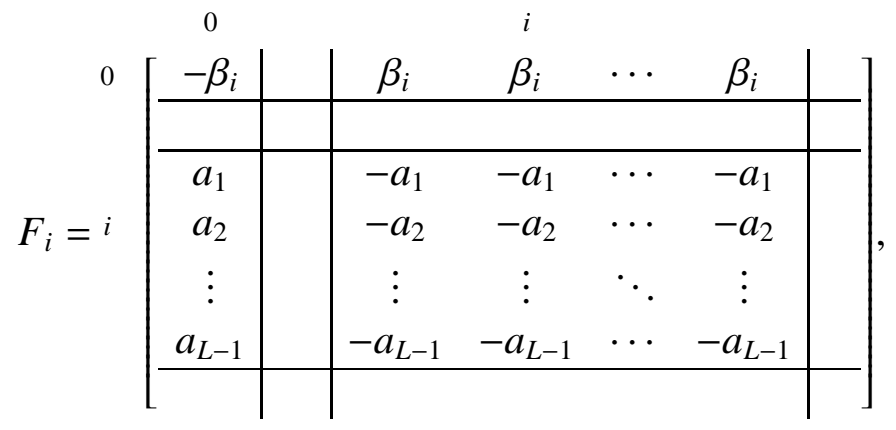




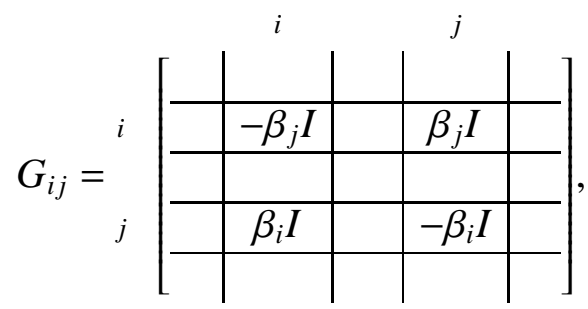

and $a_{n}=\alpha_{n}-\gamma_{n}$ and $b_{i, n}=\sum_{j \neq i} \beta_{j}-\gamma_{n}$; the symbol $I$ denotes the identity matrix of size $L-1$. We wrote a square matrix $M$ of size $N(L-1)+1$ as

$$
\left.M=\begin{array}{c|c|c|c}
0 & 1 & \multicolumn{1}{c}{} \\
1 \\
M_{00} & M_{01} & \cdots & M_{0 N} \\
\hline M_{10} & M_{11} & \cdots & M_{1 N} \\
\hline \vdots & \vdots & \ddots & \vdots \\
\hline M_{N 0} & M_{N 1} & \cdots & M_{N N}
\end{array}\right]
$$

with dividing it into $(N+1)^{2}$ blocks so that $M_{00}$ becomes a scalar, $M_{0 j}(j \neq 0)$ and $M_{i 0}(i \neq 0)$ row and column $(L-1)$-vectors, respectively, and $M_{i j}(i, j \neq 0)$ a square matrix of size $L-1$.

The linear Pffafian system (2.5), or (2.6), is of rank $N(L-1)+1$ and the integrals

$$
\vec{y}\left(x ; \Delta_{0}\right) \quad \text { and } \quad \vec{y}\left(x ; \Delta_{n}^{(i)}\right) \quad\left(\begin{array}{c}
1 \leq i \leq N \\
1 \leq n \leq L-1
\end{array}\right)
$$

provide a fundamental system of solutions. In particular, $\vec{y}\left(x ; \Delta_{0}\right)$ is the unique holomorphic solution at $0 \in \mathbb{C}^{N}$ up to multiplication by constants; it is expressible in terms of the hypergeometric function $F_{L, N}(\alpha, \beta, \gamma ; x)$ according to the integral representation (see Proposition 2.1) as

$$
\begin{aligned}
& y_{0}=c F_{L, N}, \quad y_{1}^{(i)}=\frac{\gamma_{1}-\alpha_{1}}{\gamma_{1}} c F_{L, N}\left(\beta_{i}+1, \gamma_{1}+1\right), \\
& y_{2}^{(i)}=\frac{\alpha_{1}\left(\gamma_{2}-\alpha_{2}\right)}{\gamma_{1} \gamma_{2}} c F_{L, N}\left(\alpha_{1}+1, \beta_{i}+1, \gamma_{1}+1, \gamma_{2}+1\right), \quad \ldots \\
& y_{n}^{(i)}=\frac{\alpha_{1} \cdots \alpha_{n-1}\left(\gamma_{n}-\alpha_{n}\right)}{\gamma_{1} \cdots \gamma_{n}} c F_{L, N}\left(\alpha_{1}+1, \ldots, \alpha_{n-1}+1, \beta_{i}+1, \gamma_{1}+1, \ldots, \gamma_{n}+1\right), \quad \ldots
\end{aligned}
$$

where $c=\prod_{k=1}^{L-1} \Gamma\left(\alpha_{k}\right) \Gamma\left(\gamma_{k}-\alpha_{k}\right) / \Gamma\left(\gamma_{k}\right)$. For notational simplicity, we used the abbreviation $F_{L, N}\left(\beta_{i}+\right.$ $\left.1, \gamma_{1}+1\right)$ to mean that among the parameters $(\alpha, \beta, \gamma)$ only the indicated ones $\beta_{i}$ and $\gamma_{1}$ are shifted by one, and so forth. We mention that the differential equations satisfied by the first element $y=y_{0}$ of $\vec{y}$ are indeed (1.3).

\subsection{Verification of Theorem 2.2}

In general, it holds for an $(L-1)$-form $\varphi$ that

$$
\frac{\partial}{\partial x_{i}} \int_{\Delta} U \varphi=\int_{\Delta} U\left(\frac{1}{U} \frac{\partial U}{\partial x_{i}} \varphi+\frac{\partial \varphi}{\partial x_{i}}\right) .
$$

Hence Theorem 2.2 is an immediate consequence of (2.4) and the following lemma. 
Lemma 2.3. Define a linear operator $\nabla_{i}(i=1, \ldots, N)$ acting on a differential form $\varphi$ by

$$
\nabla_{i} \varphi=\frac{1}{U} \frac{\partial U}{\partial x_{i}} \varphi+\frac{\partial \varphi}{\partial x_{i}}
$$

The rational $(L-1)$-forms $\varphi_{0}$ and $\varphi_{n}^{(i)}$ satisfy the relations

$$
\begin{aligned}
\left(x_{i}-1\right) \nabla_{i} \varphi_{0}= & \beta_{i}\left(-\varphi_{0}+\sum_{m=1}^{L-1} \varphi_{m}^{(i)}\right) \\
\left(x_{i}-x_{j}\right) \nabla_{i} \varphi_{n}^{(j)}= & \beta_{i}\left(\varphi_{n}^{(i)}-\varphi_{n}^{(j)}\right), \\
x_{i} \nabla_{i} \varphi_{n}^{(i)} \equiv & -\alpha_{n} \varphi_{n}^{(i)}+\left(\gamma_{n}-\alpha_{n}\right) \sum_{m=n+1}^{L-1} \varphi_{m}^{(i)}+\frac{\gamma_{n}-\alpha_{n}}{x_{i}-1}\left(-\varphi_{0}+\sum_{m=1}^{L-1} \varphi_{m}^{(i)}\right) \\
& +\sum_{\substack{j=1 \\
j \neq i}}^{N} \frac{\beta_{j} x_{j}}{x_{i}-x_{j}}\left(\varphi_{n}^{(j)}-\varphi_{n}^{(i)}\right) \quad\left(\operatorname{modulo} \nabla_{\omega} \Omega^{L-2}(* D)\right) .
\end{aligned}
$$

Proof. We will use the notation

$$
\begin{aligned}
\frac{\mathrm{d} t}{-} & =\mathrm{d} t_{1} \wedge \cdots \wedge \mathrm{d} t_{L-1}, \\
* \mathrm{~d} t_{j} & =(-1)^{j-1} \mathrm{~d} t_{1} \wedge \cdots \wedge \widehat{\mathrm{d} t_{j}} \wedge \cdots \wedge \mathrm{d} t_{L-1} ;
\end{aligned}
$$

therefore, $\mathrm{d} t_{j} \wedge * \mathrm{~d} t_{j}=\underline{\mathrm{d} t}$. We abbreviate $\prod_{k=1}^{L-1}$ and $\prod_{\substack{k=1 \\ k \neq n}}^{L-1}$ respectively to $\prod_{k}$ and $\prod_{k \neq n}$, and so forth. From the definition

$$
\varphi_{0}=\frac{\underline{\mathrm{d} t}}{t_{L-1} \prod_{k}\left(t_{k-1}-t_{k}\right)}, \quad \varphi_{n}^{(i)}=\frac{\underline{\mathrm{d} t}}{t_{L-1}\left(1-x_{i} t_{L-1}\right) \prod_{k \neq n}\left(t_{k-1}-t_{k}\right)},
$$

it is readily seen that

$$
\begin{aligned}
\sum_{m=n+1}^{L-1} \varphi_{m}^{(i)} & =\frac{t_{n}-t_{L-1}}{t_{L-1}\left(1-x_{i} t_{L-1}\right) \prod_{k}\left(t_{k-1}-t_{k}\right)} \frac{\mathrm{d} t}{x_{i}-1} \quad(0 \leq n \leq L-2), \\
-\varphi_{0} & +\sum_{m=1}^{L-1} \varphi_{m}^{(i)}=\frac{\mathrm{d} t .}{\left(1-x_{i} t_{L-1}\right) \prod_{k}\left(t_{k-1}-t_{k}\right)}
\end{aligned}
$$

Since $\varphi_{0}$ does not depend on $x_{i}$ it follows that

$$
\nabla_{i} \varphi_{0}=\frac{1}{U} \frac{\partial U}{\partial x_{i}} \varphi_{0}=\frac{\beta_{i} t_{L-1}}{1-x_{i} t_{L-1}} \varphi_{0}=\frac{\beta_{i} \underline{\mathrm{d} t}}{\left(1-x_{i} t_{L-1}\right) \prod_{k}\left(t_{k-1}-t_{k}\right)},
$$

which coincides with (2.7a) according to (2.10). Likewise (2.7b) can be verified as

$$
\begin{aligned}
\nabla_{i} \varphi_{n}^{(j)} & =\frac{\beta_{i} \underline{\mathrm{d} t}}{\left(1-x_{i} t_{L-1}\right)\left(1-x_{j} t_{L-1}\right) \prod_{k \neq n}\left(t_{k-1}-t_{k}\right)} \\
& =\frac{\beta_{i}}{x_{i}-x_{j}}\left(\frac{1}{1-x_{i} t_{L-1}}-\frac{1}{1-x_{j} t_{L-1}}\right) \frac{\underline{\mathrm{d}}}{t_{L-1} \prod_{k \neq n}\left(t_{k-1}-t_{k}\right)} \\
& =\frac{\beta_{i}}{x_{i}-x_{j}}\left(\varphi_{n}^{(i)}-\varphi_{n}^{(j)}\right) .
\end{aligned}
$$


It is difficult to calculate directly $\nabla_{i} \varphi_{n}^{(i)}$ because $\varphi_{n}^{(i)}$ depends on $x_{i}$. So we first prepare an appropriate coboundary, by which we eliminate the $x_{i}$ dependence of $\varphi_{n}^{(i)}$ modulo $\nabla_{\omega} \Omega^{L-2}(* D)$. Consider the rational $(L-2)$-form

$$
\begin{aligned}
\Omega^{L-2}(* D) \ni \xi_{n} & =\prod_{k \neq n} \mathrm{~d} \log \left(t_{k-1}-t_{k}\right) \wedge \\
& =\frac{(-1)^{L+n-1}}{\prod_{k \neq n}\left(t_{k-1}-t_{k}\right)} \sum_{j=n}^{L-1} * \mathrm{~d} t_{j} .
\end{aligned}
$$

Its covariant derivative reads (recall (2.3))

$$
\begin{aligned}
\nabla_{\omega} \xi_{n}=( & -1)^{L+n}\left(\frac{\gamma_{n}-\alpha_{n}}{\prod_{k}\left(t_{k-1}-t_{k}\right)}-\frac{\alpha_{L-1}}{t_{L-1} \prod_{k \neq n}\left(t_{k-1}-t_{k}\right)}\right. \\
& \left.\quad-\sum_{j=1}^{N} \frac{\beta_{j} x_{j}}{\left(1-x_{j} t_{L-1}\right) \prod_{k \neq n}\left(t_{k-1}-t_{k}\right)}+\sum_{j=n}^{L-2} \frac{\gamma_{j+1}-\alpha_{j}}{t_{j} \prod_{k \neq n}\left(t_{k-1}-t_{k}\right)}\right) \underline{\mathrm{d} t} .
\end{aligned}
$$

Hence

$$
\begin{aligned}
\sum_{j=1}^{N} \beta_{j} \varphi_{n}^{(j)} & =\sum_{j=1}^{N} \beta_{j}\left(\frac{1}{t_{L-1} \prod_{k \neq n}\left(t_{k-1}-t_{k}\right)}+\frac{x_{j}}{\left(1-x_{j} t_{L-1}\right) \prod_{k \neq n}\left(t_{k-1}-t_{k}\right)}\right) \underline{\mathrm{d} t} \\
& \equiv\left(\frac{\gamma_{n}-\alpha_{n}}{\prod_{k}\left(t_{k-1}-t_{k}\right)}+\frac{\sum_{j=1}^{N} \beta_{j}-\alpha_{L-1}}{t_{L-1} \prod_{k \neq n}\left(t_{k-1}-t_{k}\right)}+\sum_{j=n}^{L-2} \frac{\gamma_{j+1}-\alpha_{j}}{t_{j} \prod_{k \neq n}\left(t_{k-1}-t_{k}\right)}\right) \underline{\mathrm{d} t}
\end{aligned}
$$

modulo $\nabla_{\omega} \xi_{n}$. Now the derivative can be calculated as

$$
\begin{aligned}
\left(\frac{x_{i}}{\beta_{i}} \nabla_{i}+1\right) \sum_{j=1}^{N} \beta_{j} \varphi_{n}^{(j)}= & \left(\frac{1}{1-x_{i} t_{L-1}}+\frac{x_{i}}{\beta_{i}} \frac{\partial}{\partial x_{i}}\right) \sum_{j=1}^{N} \beta_{j} \varphi_{n}^{(j)} \\
\equiv & \left(\frac{\gamma_{n}-\alpha_{n}}{\left(1-x_{i} t_{L-1}\right) \prod_{k}\left(t_{k-1}-t_{k}\right)}+\frac{\sum_{j=1}^{N} \beta_{j}-\alpha_{L-1}}{t_{L-1}\left(1-x_{i} t_{L-1}\right) \prod_{k \neq n}\left(t_{k-1}-t_{k}\right)}\right. \\
& \left.\quad+\sum_{j=n}^{L-2} \frac{\gamma_{j+1}-\alpha_{j}}{t_{j}\left(1-x_{i} t_{L-1}\right) \prod_{k \neq n}\left(t_{k-1}-t_{k}\right)}\right) \underline{\mathrm{d} t .}
\end{aligned}
$$

Applying (2.8), (2.10) and Lemma 2.4 below, we thus arrive at

$$
\left(\frac{x_{i}}{\beta_{i}} \nabla_{i}+1\right) \sum_{j=1}^{N} \beta_{j} \varphi_{n}^{(j)} \equiv \frac{\gamma_{n}-\alpha_{n}}{x_{i}-1}\left(-\varphi_{0}+\sum_{m=1}^{L-1} \varphi_{m}^{(i)}\right)+\left(\sum_{j=1}^{N} \beta_{j}-\alpha_{n}\right) \varphi_{n}^{(i)}+\left(\gamma_{n}-\alpha_{n}\right) \sum_{m=n+1}^{L-1} \varphi_{m}^{(i)},
$$

which establishes (2.7c by virtue of (2.7b). The proof of the lemma is complete.

Lemma 2.4. One has

$$
\sum_{j=n}^{L-2} \frac{\gamma_{j+1}-\alpha_{j}}{t_{j}\left(1-x_{i} t_{L-1}\right) \prod_{k \neq n}\left(t_{k-1}-t_{k}\right)} \frac{\mathrm{d} t}{\underline{ }} \equiv\left(\alpha_{L-1}-\alpha_{n}\right) \varphi_{n}^{(i)}+\left(\gamma_{n}-\alpha_{n}\right) \sum_{m=n+1}^{L-1} \varphi_{m}^{(i)}
$$

modulo $\nabla_{\omega} \Omega^{L-2}(* D)$. 
Proof. Taking partial fraction decomposition yields

$$
\begin{aligned}
& \sum_{j=n}^{L-2} \frac{\gamma_{j+1}-\alpha_{j}}{t_{j}\left(1-x_{i} t_{L-1}\right) \prod_{k \neq n}\left(t_{k-1}-t_{k}\right)} \frac{\mathrm{d} t}{1} \\
& =\frac{1}{t_{L-1}\left(1-x_{i} t_{L-1}\right)} \sum_{j=n}^{L-2}\left(\gamma_{j+1}-\alpha_{j}\right)\left(\frac{1}{\prod_{k \neq n}\left(t_{k-1}-t_{k}\right)}-\frac{1}{t_{j}} \sum_{m=j+1}^{L-1} \frac{1}{\prod_{k \neq n, m}\left(t_{k-1}-t_{k}\right)}\right) \underline{\mathrm{d} t} \\
& =\sum_{j=n}^{L-2}\left(\gamma_{j+1}-\alpha_{j}\right) \varphi_{n}^{(i)}-\frac{1}{t_{L-1}\left(1-x_{i} t_{L-1}\right)} \sum_{j=n}^{L-2} \sum_{m=j+1}^{L-1} \frac{\gamma_{j+1}-\alpha_{j}}{t_{j} \prod_{k \neq n, m}\left(t_{k-1}-t_{k}\right)} \frac{\mathrm{d} t .}{}
\end{aligned}
$$

On the other hand, we consider the $(L-2)$-form

$$
\begin{aligned}
\Omega^{L-2}(* D) \ni \psi_{n, m} & =\left(\prod_{k \neq n, m} \mathrm{~d} \log \left(t_{k-1}-t_{k}\right) \wedge\right) \mathrm{d} t_{L-1} \\
& =\frac{(-1)^{n+m-1}}{\prod_{k \neq n, m}\left(t_{k-1}-t_{k}\right)} \sum_{j=n}^{m-1} * \mathrm{~d} t_{j}
\end{aligned}
$$

for $n<m$, of which the covariant derivative reads

$$
\nabla_{\omega} \psi_{n, m}=(-1)^{n+m}\left(\sum_{j=n}^{m-1} \frac{\gamma_{j+1}-\alpha_{j}}{t_{j} \prod_{k \neq n, m}\left(t_{k-1}-t_{k}\right)}+\frac{\gamma_{n}-\alpha_{n}}{\prod_{k \neq m}\left(t_{k-1}-t_{k}\right)}-\frac{\gamma_{m}-\alpha_{m}}{\prod_{k \neq n}\left(t_{k-1}-t_{k}\right)}\right) \underline{\mathrm{d} t} .
$$

Observe that $\nabla_{\omega} \psi_{m, n}$ still remains a coboundary if we multiply it by any rational function $g \in$ $\Omega^{0}(* D)$ such that $\partial g / \partial t_{k}=0(\forall k \neq L-1)$. In particular, by choosing $g=t_{L-1}{ }^{-1}\left(1-x_{i} t_{L-1}\right)^{-1}$, we have

$$
\frac{1}{t_{L-1}\left(1-x_{i} t_{L-1}\right)} \sum_{j=n}^{m-1} \frac{\gamma_{j+1}-\alpha_{j}}{t_{j} \prod_{k \neq n, m}\left(t_{k-1}-t_{k}\right)} \mathrm{d} t+\left(\gamma_{n}-\alpha_{n}\right) \varphi_{m}^{(i)}-\left(\gamma_{m}-\alpha_{m}\right) \varphi_{n}^{(i)} \equiv 0 .
$$

Summation over $m=n+1, \ldots, L-1$ of this formula entails

$$
\frac{1}{t_{L-1}\left(1-x_{i} t_{L-1}\right)} \sum_{m=n+1}^{L-1} \sum_{j=n}^{m-1} \frac{\gamma_{j+1}-\alpha_{j}}{t_{j} \prod_{k \neq n, m}\left(t_{k-1}-t_{k}\right)} \mathrm{d} t+\left(\gamma_{n}-\alpha_{n}\right) \sum_{m=n+1}^{L-1} \varphi_{m}^{(i)}-\sum_{m=n+1}^{L-1}\left(\gamma_{m}-\alpha_{m}\right) \varphi_{n}^{(i)} \equiv 0 .
$$

Substituting the above into (2.12), we verify the desired result (2.11).

\section{Hypergeometric solution of Hamiltonian system $\mathcal{H}_{L, N}$}

In this section we first review Lax formalism of $\mathcal{H}_{L, N}$ following [15]. Under a certain condition of parameters $\mathcal{H}_{L, N}$ admits particular solutions such that the associated Fuchsian system becomes reducible. These solutions are governed by the Pfaffian system derived in the previous section and, thereby, expressible in terms of the hypergeometric function. 


\subsection{Lax formalism of $\mathcal{H}_{L . N}$}

We begin with a brief review of Lax formalism of $\mathcal{H}_{L, N}$. Consider an $L \times L$ Fuchsian system

$$
\frac{\partial \Phi}{\partial z}=A \Phi=\sum_{i=0}^{N+1} \frac{A_{i}}{z-u_{i}} \Phi
$$

with $N+3$ regular singularities $\left\{u_{0}=1, u_{1}, \ldots, u_{N}, u_{N+1}=0, u_{N+2}=\infty\right\} \subset \mathbb{P}^{1}$, of which the characteristic exponents at each singularity $z=u_{i}$, i.e., the eigenvalues of each residue matrix $A_{i}$, are listed in the following table (Riemann scheme):

\begin{tabular}{cc}
\hline Singularity & Exponents \\
\hline$u_{i}(0 \leq i \leq N)$ & $\left(-\theta_{i}, 0, \ldots, 0\right)$ \\
$u_{N+1}=0$ & $\left(e_{0}, e_{1}, \ldots, e_{L-1}\right)$ \\
$u_{N+2}=\infty$ & $\left(\kappa_{0}-e_{0}, \kappa_{1}-e_{1}, \ldots, \kappa_{L-1}-e_{L-1}\right)$ \\
\hline
\end{tabular}

We can, and will, normalize the exponents as $\operatorname{tr} A_{N+1}=\sum_{n=0}^{L-1} e_{n}=(L-1) / 2$ without loss of generality. Assume $\sum_{n=0}^{L-1} \kappa_{n}=\sum_{i=0}^{N} \theta_{i}$ (Fuchs' relation). Such Fuchsian systems as above then turn out to constitute a $2 N(L-1)$-dimensional family and, actually, can be written in terms of the accessory parameters $b_{n}^{(i)}$ and $c_{n}^{(i)}$ in the following way:

$$
\begin{aligned}
A_{i} & ={ }^{\mathrm{T}}\left(b_{0}^{(i)}, b_{1}^{(i)}, \ldots, b_{L-1}^{(i)}\right) \cdot\left(c_{0}^{(i)}, c_{1}^{(i)}, \ldots, c_{L-1}^{(i)}\right) \quad(0 \leq i \leq N), \\
A_{N+1} & =\left(\begin{array}{cccc}
e_{0} & w_{0,1} & \cdots & w_{0, L-1} \\
& e_{1} & \ddots & \vdots \\
& & \ddots & w_{L-2, L-1} \\
& & & e_{L-1}
\end{array}\right),
\end{aligned}
$$

where $c_{0}^{(i)}=1$ and $w_{m, n}=-\sum_{i=0}^{N} b_{m}^{(i)} c_{n}^{(i)}$. We thus find the relations

$$
\left(\operatorname{tr} A_{i}=\right) \sum_{n=0}^{L-1} b_{n}^{(i)} c_{n}^{(i)}=-\theta_{i} \quad \text { and } \quad \sum_{i=0}^{N} b_{n}^{(i)} c_{n}^{(i)}=-\kappa_{n},
$$

the latter of which comes from the diagonal entries of the lower triangular matrix $A_{N+2}=-\sum_{i=0}^{N+1} A_{i}$. Since $A_{N+1}$ and $A_{N+2}$ are triangular, there still remains the degree of freedom of a similarity transformation by a diagonal matrix. Consequently, the essential number of the accessory parameters is confirmed to be $2 N(L-1)$. In fact, they can be realized by the canonical variables $q_{n}^{(i)}$ and $p_{n}^{(i)}$ of $\mathcal{H}_{L, N}$; see (3.5) below.

The isomonodromic family of Fuchsian systems of the form (3.1) is described by the integrability condition of the extended linear system, i.e., (3.1) itself and its deformation equations

$$
\frac{\partial \Phi}{\partial u_{i}}=B_{i} \Phi, \quad B_{i}=\frac{A_{i}}{u_{i}-z}-\frac{1}{u_{i}}\left(\begin{array}{ccc}
-\frac{\theta_{i}}{L} & & \\
& \ddots & \\
* & & -\frac{\theta_{i}}{L}
\end{array}\right) \quad(1 \leq i \leq N) .
$$

Here the lower triangular part $(*)$ of the second term is exactly the same as $A_{i}$. 
Theorem 3.1 (See [15]). The integrability condition

$$
\frac{\partial A}{\partial u_{i}}-\frac{\partial B_{i}}{\partial z}+\left[A, B_{i}\right]=0
$$

of (3.1) and (3.3) is equivalent to the polynomial Hamiltonian system $\mathcal{H}_{L, N}$, (1.1), via the change of variables

$$
x_{i}=\frac{1}{u_{i}}, \quad q_{n}^{(i)}=\frac{c_{n}^{(i)}}{c_{n}^{(0)}} \quad \text { and } \quad p_{n}^{(i)}=-b_{n}^{(i)} c_{n}^{(0)} .
$$

\subsection{Particular solution of $\mathcal{H}_{L, N}$}

Suppose that $\kappa_{0}-\sum_{i=1}^{N} \theta_{i}=0$. This condition enables us to restrict $b_{n}^{(i)}$ and $c_{n}^{(i)}$ to the subvariety

$$
\begin{aligned}
& b_{0}^{(0)}=0, \quad b_{0}^{(i)}=-\theta_{i} \quad(i \neq 0), \\
& c_{1}^{(0)}=\cdots=c_{L-1}^{(0)} \quad \text { and } \quad c_{n}^{(i)}=0 \quad(i \neq 0, n \neq 0)
\end{aligned}
$$

while keeping consistency of the linear system (3.1) and (3.3). Notice here that this restriction amounts to $q_{n}^{(i)}=0$. In view of (3.2) we see that the matrices $A_{i}$ and $B_{i}$ can be parameterized by the $N(L-1)+1$ variables $f:=1 / c_{1}^{(0)}=\cdots=1 / c_{L-1}^{(0)}$ and $b_{n}^{(i)}(i \neq 0, n \neq 0)$. It actually follows that

$$
\begin{aligned}
& A_{0}=\left(\begin{array}{cccc}
0 & 0 & \cdots & 0 \\
-\kappa_{1} f & -\kappa_{1} & \cdots & -\kappa_{1} \\
\vdots & \vdots & \ddots & \vdots \\
-\kappa_{L-1} f & -\kappa_{L-1} & \cdots & -\kappa_{L-1}
\end{array}\right), \quad A_{i}=\left(\begin{array}{cccc}
-\theta_{i} & 0 & \cdots & 0 \\
b_{1}^{(i)} & 0 & \cdots & 0 \\
\vdots & \vdots & \ddots & \vdots \\
b_{L-1}^{(i)} & 0 & \cdots & 0
\end{array}\right) \quad(1 \leq i \leq N), \\
& A_{N+1}=\left(\begin{array}{cccccc}
e_{0} & 0 & 0 & 0 & \cdots & 0 \\
& e_{1} & \kappa_{1} & \kappa_{1} & \cdots & \kappa_{1} \\
& & e_{2} & \kappa_{2} & \cdots & \kappa_{2} \\
& & & e_{3} & \ddots & \vdots \\
& & & & \ddots & \kappa_{L-2} \\
& & & & & e_{L-1}
\end{array}\right),
\end{aligned}
$$

therefore, (3.1) is clearly reducible. On the other hand, we have

$$
B_{i}=\frac{\theta_{i}}{L u_{i}}\left(\begin{array}{cccc}
1-L & & & \\
& 1 & & \\
& & \ddots & \\
& & & 1
\end{array}\right)+\frac{z}{u_{i}\left(u_{i}-z\right)}\left(\begin{array}{cccc}
-\theta_{i} & 0 & \cdots & 0 \\
b_{1}^{(i)} & 0 & \cdots & 0 \\
\vdots & \vdots & \ddots & \vdots \\
b_{L-1}^{(i)} & 0 & \cdots & 0
\end{array}\right) .
$$


Observe that in this situation only the $(n, 0)$-entries $(1 \leq n \leq L-1)$ of the matrix equation (3.4) are nontrivial. In fact,

$$
\begin{aligned}
\left(\frac{\partial A}{\partial u_{i}}-\frac{\partial B_{i}}{\partial z}\right)_{n, 0}= & \frac{-\kappa_{n}}{z-1} \frac{\partial f}{\partial u_{i}}+\sum_{j=1}^{N} \frac{1}{z-u_{j}} \frac{\partial b_{n}^{(j)}}{\partial u_{i}}, \\
{\left[A, B_{i}\right]_{n, 0}=} & \frac{\kappa_{n}}{u_{i}(z-1)\left(z-u_{i}\right)}\left(-\theta_{i} u_{i} f+z \sum_{m=1}^{L-1} b_{m}^{(i)}\right) \\
& +\frac{1}{u_{i}\left(z-u_{i}\right)}\left(\left(e_{0}-e_{n}\right) b_{n}^{(i)}-\kappa_{n} \sum_{m=n+1}^{L-1} b_{m}^{(i)}\right) \\
& +\frac{1}{u_{i}\left(z-u_{i}\right)} \sum_{j=1}^{N} \frac{\theta_{i} b_{n}^{(j)} u_{i}-\theta_{j} b_{n}^{(i)} z}{z-u_{j}} .
\end{aligned}
$$

Residue calculus at $z=1, z=u_{j}(j \neq i)$ and $z=u_{i}$ yields the system of linear differential equations for unknowns $f$ and $b_{n}^{(i)}$ :

$$
\begin{aligned}
u_{i}\left(1-u_{i}\right) \frac{\partial f}{\partial u_{i}}= & -u_{i} \theta_{i} f+\sum_{m=1}^{L-1} b_{m}^{(i)}, \\
\left(u_{i}-u_{j}\right) \frac{\partial b_{n}^{(j)}}{\partial u_{i}}= & \theta_{i} b_{n}^{(j)}-\frac{u_{j}}{u_{i}} \theta_{j} b_{n}^{(i)}, \\
\frac{\partial b_{n}^{(i)}}{\partial u_{i}}= & \frac{1}{u_{i}}\left(\left(e_{n}-e_{0}+\theta_{i}\right) b_{n}^{(i)}+\kappa_{n} \sum_{m=n+1}^{L-1} b_{m}^{(i)}\right)+\frac{\kappa_{n}}{u_{i}-1}\left(\theta_{i} f-\sum_{m=1}^{L-1} b_{m}^{(i)}\right) \\
& -\sum_{\substack{j=1 \\
j \neq i}}^{N} \frac{\theta_{i} b_{n}^{(j)}-\theta_{j} b_{n}^{(i)}}{u_{i}-u_{j}} .
\end{aligned}
$$

If we apply the change of variables

$$
x_{i}=\frac{1}{u_{i}}, \quad y_{0}=\frac{f}{\prod_{j=1}^{N} u_{j}^{\theta_{j}}} \quad \text { and } \quad y_{n}^{(i)}=\frac{b_{n}^{(i)}}{\theta_{i} \prod_{j=1}^{N} u_{j}^{\theta_{j}}},
$$

then (3.6) is converted into the Pfaffian system for $F_{L, N}$ (see (2.5) in Theorem 2.2) with $\alpha_{n}=e_{n}-e_{0}$, $\beta_{n}=-\theta_{n}$ and $\gamma_{n}=e_{n}-e_{0}-\kappa_{n}$. Combining this fact with Theorem 3.1, we finally arrive at the

Theorem 3.2. When $\kappa_{0}-\sum_{i=1}^{N} \theta_{i}=0$, the Hamiltonian system $\mathcal{H}_{L, N}$ admits a particular solution of the form

$$
q_{n}^{(i)}=0, \quad p_{n}^{(i)}=-\theta_{i} \frac{y_{n}^{(i)}}{y_{0}} \quad\left(\begin{array}{c}
1 \leq i \leq N \\
1 \leq n \leq L-1
\end{array}\right)
$$

where $\left\{y_{0}, y_{n}^{(i)}\right\}$ is an arbitrary solution of the linear Pfaffian system, (2.5) or (2.6), with

$$
\alpha_{n}=e_{n}-e_{0}, \quad \beta_{n}=-\theta_{n}, \quad \gamma_{n}=e_{n}-e_{0}-\kappa_{n} .
$$


Remark 3.3. We already know that $y_{0}$ is a solution of the hypergeometric equation (1.3). Moreover, it is possible to write all the other elements $y_{n}^{(i)}$ as linear combinations of derivatives of $y_{0}$. In fact, we can carry out it by the differential operators appearing in the contiguity relations for the hypergeometric series $F_{L, N}$; cf. Sect. 2 and the appendix below.

Remark 3.4. Particular solutions of the Schlesinger system for the case of a general spectral type have been studied by Dubrovin-Mazzocco [3]. For instance they showed that if the monodromy group of the associated Fuchsian system is triangular, then the Schlesinger system can be solved in terms of solutions of linear differential equations (that are generally inhomogeneous).

\section{A Contiguity relations for $F_{L, N}$}

In this appendix we provide a table of the contiguity relations for $F=F_{L, N}(\alpha, \beta, \gamma ; x)$. We shall use again such an abbreviation as $F\left(\alpha_{n}+1\right)$ to represent the same function as $F$ except increasing the indicated parameter $\alpha_{n}$ by one. Recall the notation (1.4) of the Eular operators.

Theorem A.1. The hypergeometric function $F=F_{L, N}(\alpha, \beta, \gamma ; x)$ satisfies the contiguity relations

$$
\begin{aligned}
& F\left(\alpha_{n}+1\right)=\frac{\mathcal{D}+\alpha_{n}}{\alpha_{n}} F, \\
& F\left(\beta_{i}+1\right)=\frac{\delta_{i}+\beta_{i}}{\beta_{i}} F, \\
& F\left(\gamma_{n}+1\right)=\frac{\gamma_{n}}{\varepsilon_{L}}\left\{\sum_{i=1}^{N} \frac{\partial}{\partial x_{i}} \prod_{\substack{k=1 \\
k \neq n}}^{L-1}\left(\mathcal{D}+\gamma_{k}-1\right)-\sum_{j=0}^{L-1} \varepsilon_{j}\left(\mathcal{D}+\gamma_{n}\right)^{L-1-j}\right\} F, \\
& F\left(\alpha_{n}-1\right)=\frac{\alpha_{n}-1}{\varepsilon_{L}^{\prime}}\left\{\sum_{i=1}^{N} x_{i}\left(\delta_{i}+\beta_{i}\right) \prod_{\substack{k=1 \\
k \neq n}}^{L-1}\left(\mathcal{D}+\alpha_{k}\right)-\sum_{j=0}^{L-1} \varepsilon_{j}^{\prime}\left(\mathcal{D}+\alpha_{n}-1\right)^{L-1-j}\right\} F, \\
& F\left(\gamma_{n}-1\right)=\frac{\mathcal{D}+\gamma_{n}-1}{\gamma_{n}-1} F \text {, } \\
& F\left(\beta_{i}+1, \beta_{j}-1\right)=\frac{\left(x_{i}-x_{j}\right) \frac{\partial}{\partial x_{i}}+\beta_{i}}{\beta_{i}} F, \\
& F\left(\alpha_{1}+1, \ldots, \alpha_{L-1}+1, \beta_{i}+1, \gamma_{1}+1, \ldots, \gamma_{L-1}+1\right)=\frac{\gamma_{1} \cdots \gamma_{L-1}}{\alpha_{1} \cdots \alpha_{L-1} \beta_{i}} \frac{\partial F}{\partial x_{i}} .
\end{aligned}
$$

Here each $\varepsilon_{j}$ denotes the $j$-th elementary symmetric polynomial in $L$ variables $\alpha_{k}-\gamma_{n}(k=$ $1, \ldots, L-1)$ and $\sum_{i=1}^{N} \beta_{i}-\gamma_{n}$, and similarly $\varepsilon_{j}^{\prime}$ does that in $\gamma_{k}-\alpha_{n}(k=1, \ldots, L-1)$ and $1-\alpha_{n}$.

Proof. Notice the formula $\delta_{i} x_{i}=x_{i}\left(\delta_{i}+1\right)$ with $x_{i}$ regarded as the operator multiplying $x_{i}$. Then (A.1), (A.2), (A.5) and (A.7) are immediate from the definition (1.2) of the hypergeometric series. By an analogue of the classical factorization method, (A.3), (A.4) and (A.6) can be obtained.

Let $\mathcal{S}=\mathcal{S}(\alpha, \beta, \gamma)$ be the linear space of solutions of the hypergeometric equation (1.3). In general, the linear operators appearing in Theorem A.1 induce isomorphisms of these spaces. For 
instance, let $H$ and $B$ be the differential operators defined by

$$
H=\sum_{i=1}^{N} \frac{\partial}{\partial x_{i}} \prod_{\substack{k=1 \\ k \neq n}}^{L-1}\left(\mathcal{D}+\gamma_{k}-1\right)-\sum_{j=0}^{L-1} \varepsilon_{j}\left(\mathcal{D}+\gamma_{n}\right)^{L-1-j}, \quad B=\mathcal{D}+\gamma_{n}
$$

cf. (A.3) and (A.5). The linear homomorphisms

$$
H: \mathcal{S} \rightarrow \mathcal{S}\left(\gamma_{n}+1\right), \quad B: \mathcal{S}\left(\gamma_{n}+1\right) \rightarrow \mathcal{S}
$$

are isomorphisms if and only if $\gamma_{n} \varepsilon_{L} \neq 0$.

Acknowledgement. The author is grateful to Masaaki Yoshida for reading carefully the first draft of this article and giving helpful comments. He has also benefited from discussions with Yousuke Ohyama and Yasuhiko Yamada.

\section{References}

[1] K. Aomoto and M. Kita, Hypergeometric Functions, Springer-Verlag Tokyo, 1994 (Japanese).

[2] P. Appell and J. Kampé de Fériet, Fonctions Hypergéométriques et Hypersphériques, Polynomes d'Hermite, Gauthier-Villars Paris, 1926 (French).

[3] B. Dubrovin and M. Mazzocco, On the reductions and classical solutions of the Schlesinger equations, in: D. Bertrand, B. Enriquez, C. Mitschi, C. Sabbah and R. Schäfke (Eds.), Differential Equations and Quantum Groups, Eur. Math. Soc., Zürich 2007, pp. 157-187.

[4] R. Fuchs, Über lineare homogene Differentialgleichungen zweiter Ordnung mit drei im Endlichen gelegenen wesentlich singulären Stellen, Math. Ann. 63 (1907), 301-321 (German).

[5] R. Garnier, Sur des équations différentielles du troisième ordre dont l'intégrale générale est uniforme et sur une classe d'équations nouvelles d'ordre supérieur dont l'intégrale générale a ses points critiques fixes, Ann. Sci. École Norm. Sup. 29 (1912), 1-126 (French).

[6] H. Kimura and K. Okamoto, On the polynomial Hamiltonian structure of the Garnier system, J. Math. Pures Appl. 63 (1984), 129-146.

[7] G. Lauricella, Sulle funzioni ipergeometriche a più variabili, Rend. Circ. Mat. Palermo 7 (1893), 111158 (Italian).

[8] J. Malmquist, Sur les équations différentielles du second ordre dont l'intégrale générale a ses points critiques fixes, Ark. Mat. Astr. Fys. 17 (1922/23), 1-89 (French).

[9] K. Okamoto, Studies on the Painlevé equations. I. Sixth Painlevé equation P ${ }_{\mathrm{VI}}$, Ann. Mat. Pura Appl. 146 (1987), 337-381.

[10] K. Okamoto and H. Kimura, On particular solutions of the Garnier systems and the hypergeometric functions of several variables, Quart. J. Math. 37 (1986), 61-80.

[11] L. Schlesinger, Über eine Klasse von Differentialsystemen beliebiger Ordnung mit festen kritischen Punkten, J. Reine Angew. Math. 141 (1912), 96-145 (German). 
[12] T. Suzuki, A particular solution of a Painlevé system in terms of the hypergeometric function ${ }_{n+1} F_{n}$, preprint (arXiv:1004.0059).

[13] J. Thomae, Ueber die höheren hypergeometrischen Reihen, insbesondere über die Reihe: $1+\frac{a_{0} a_{1} a_{2}}{1 . b_{1} b_{2}} x+$ $\frac{a_{0}\left(a_{0}+1\right) a_{1}\left(a_{1}+1\right) a_{2}\left(a_{2}+1\right)}{1.2 . b_{1}\left(b_{1}+1\right) b_{2}\left(b_{2}+1\right)} x^{2}+\cdots$, Math. Ann. 2 (1870), 427-444 (German).

[14] T. Tsuda, Universal characters and an extension of the KP hierarchy, Comm. Math. Phys. 248 (2004), 501-526.

[15] T. Tsuda, UC hierarchy and monodromy preserving deformation, preprint (MI Preprint Series, Kyushu University, MI2010-7; available also from arXiv:1007.3450).

[16] M. Yoshida, Hypergeometric Functions, My Love: Modular Interpretations of Configuration Spaces Friedrich Vieweg \& Sohn, Braunschweig, 1997. 